\title{
REPRESENTATION OF SOLUTIONS OF LINEAR INHOMOGENEOUS CAPUTO FRACTIONAL DIFFERENTIAL EQUATION WITH CONTINUOUS VARIABLE COEFFICIENT BY GREEN FUNCTION
}

\author{
Sunae PaK, Huichol Choi And KinAm Sin
}

Abstract. In this paper, the representation of solution to linear inhomogeneous Caputo fractional differential equation with continuous variable coefficient has been considered by using Green function. We have proved that the solution of linear inhomogeneous equations with homogeneous initial condition can be represented by using classical Green function, generalized Green function and modified Green function.

Mathematics subject classification (2010): 26A33, 34A08.

Keywords and phrases: Linear inhomogeneous equations, Caputo fractional differential equations, continuous variable coefficients, Green function.

\section{REFERENCES}

[1] M. KIM, Fractional differential equations and Applications, Kim Il Sung University Press, Juche 95, 2006.

[2] S. PAK, M. KIM, Existence and Uniqueness of the Solution to a Nonlinear Differential equation with Caputo Fractional Derivative in the Space of Continuously Differentiable Functions, KISU-MATH2012-E-R-005, Faculty of mathematics, Kim Il Sung University, arXiv: 1208.2107 [math-ph], (2012), pp. 1-9.

[3] S. PAK, M. Kim, H. O, Representation of Solution of linear Homogeneous Caputo Fractional Differential Equation with Continuous Variable Coefficients, KISU-MATH-2013-E-R-006, Faculty of mathematics, Kim Il Sung University, arXiv: 1305.3193 [math.CA], (2013), pp. 1-22.

[4] A. A. KilBAS, Theory and applications of fractional differential equations, North-Hell and Math. Studies. 204, (2006), 314-350.

[5] I. Podlubny, Analytical solution of linear differential equation of the fractional order, Comput. And Appl. Math., (1994), 102-106.

[6] Y. Hu, Y. Luo, Z. Lu, Analytical solution of the linear fractional differential equation by Adomian decomposition method, J. Comput. And appl. Math., 215, (2008), pp. 220-229.

[7] B. Bonilla, M. Rivero, J. J. Trujillo, On system of linear fractional differential equation with constant coefficients, Appl. Math. and comput. 187 (2007), 68-78.

[8] A. A. Kilbas, M. Rivero, L. RodrigueZ-Germa, $\alpha$-Analytic solutions of some linear fractional differential equations with variable coefficients, Appl. Math. and comput., 187 (2007), 239-249.

[9] S. R. Umarov, E. M. Saidamatov, A generalization of Duhamel's Principle for differential equations of fractional order, Dokl. math., 75, 1 (2007), 94-96.

[10] S. R. UMAROV, On fractional Duhamel's principle and its applications, Journal of Differential Equations, 252 (2012), 5217-5234. 